\title{
ESTRUTURA POPULACIONAL E ANÁLISE FITOQUÍMICA DE Metrodorea flavida K. Krause (RUTACEAE) EM DOIS FRAGMENTOS FLORESTAIS DO MUNICÍPIO DE ALTA FLORESTA, MATO GROSSO
}

Aline Gonçalves Spletozer ${ }^{1}$, Célia Regina Araújo Soares - Lopes ${ }^{2}$, Mayara Peron Pereira $^{2}$, Lucirene Rodrigues ${ }^{3}$, Cleiton Rosa dos Santos ${ }^{1}$

${ }^{1}$ Graduandos do Curso de Engenharia Florestal, Universidade do Estado de Mato Grosso - UNEMAT, Campus de Alta Floresta - MT, e-mail: alinespletozer_afmt@hotmail.com

${ }^{2}$ Professora da Faculdade de Ciências Biológicas e Agrárias, Universidade do

Estado de Mato Grosso, Campus de Alta Floresta - UNEMAT

${ }^{3}$ Mestre em Ecologia e Conservação, Universidade do Estado de Mato Grosso, Campus Nova Xavantina - MT

Recebido em: 08/09/2015 - Aprovado em: 14/11/2015 - Publicado em: 01/12/2015 DOI: http://dx.doi.org/10.18677/Enciclopedia_Biosfera_2015_153

\section{RESUMO}

Os fragmentos florestais apresentam-se cada vez mais importantes como representantes da vegetação nativa, sendo de grande relevância estudos que demonstrem a estrutura e os potenciais das espécies florestais. Assim, objetivou-se descrever a estrutura populacional de $M$. flavida em dois fragmentos florestais de Alta Floresta, e analisar qualitativamente a presença de metabólitos secundários. Para o levantamento estrutural foram implantadas 5 parcelas de $10 \times 100 \mathrm{~m}$ em cada fragmento, mensurando-se o diâmetro e a altura de todos os indivíduos com CAP $\geq$ $30 \mathrm{~cm}$. A triagem fitoquímica foi realizada com reagentes específicos para as classes de taninos, alcalóides, flavonóides e saponinas. M. flavida apresentou densidade de 84 e 32 ind. ha- ${ }^{1}$, nos fragmentos I e II, respectivamente, com indivíduos nas menores classes de diâmetro e classes intermediárias de altura. A distribuição espacial dos indivíduos foi regular para o fragmento I e aleatório para o fragmento II. Os testes fitoquímicos foram positivos para taninos, alcalóides e saponinas no fragmento I e II, e flavonóides apenas no fragmento II. A espécie apresentou elevado número de indivíduos jovens com caráter promissor para a medicina.

PALAVRAS-CHAVE: Compostos bioativos, Dados estruturais, Espécie arbórea

\section{POPULATION STRUCTURE AND ANALYSIS PHYTOCHEMISTRY OF Metrodorea flavida K.KRAUSE IN TWO FOREST FRAGMENTS IN ALTA FLORESTA, MATO GROSSO}

\section{ABSTRACT}

The forest fragments have become increasingly more important as representatives of native vegetation, these studies are very relevant because they show the structure and the potential of forest species. The aim was to describe the population structure of $M$. flavida in two forest fragments in Alta Floresta, and to analyze qualitatively the presence of secondary metabolites. For structural survey 5 plots of $10 \times 100$ m were established in each fragment to measure diameter and height of all tree with CAP $\geq$ 
$30 \mathrm{~cm}$. The phytochemistry screening was held with the specific reagents for the tannins, alkaloids, flavonoids and saponins classes. M. flavida presented density of 84 and 32 ind. ha-1, in the fragments I and II, respectively, with individuals in the smaller diameter classes and intermediate height classes. The spatial distribution of individuals was regular to fragment I and random to fragment II. Phytochemicals tests were positive to tannin, alkaloid and saponin in fragment I and II, just flavonoid to fragment II. The species showed high numbers of young trees and promising character for medicine.

KEYWORDS: Bioactive compounds, Tree species, Structural data

\section{INTRODUÇÃO}

A parte sul da floresta amazônica está fortemente ameaçada pela pressão antrópica, em face da substituição de suas paisagens naturais pela ocupação e utilização do homem (KUNZ et al., 2010). Esse fato denota o valor dos fragmentos florestais como representantes das florestas virgens originais (PAULA et al., 2015).

Estudos que descrevem a estrutura populacional de espécies arbóreas em fragmentos florestais são importantes, pois fornecem informações a respeito da ocupação dos indivíduos no ambiente e permitem inferências em relação às características ecológicas das espécies, auxiliando assim na elaboração de planos que visem a sua conservação e uso sustentável (SOUZA et al., 2009; CASTRO \& CARVALHO, 2014).

Além disso, estudos estruturais contribuem para definir o padrão de distribuição espacial dos indivíduos de uma população de acordo com as condições ambientais do remanescente que ocupa (BERNASOL e LIMA-RIBEIRO, 2010). Assim, conhecer a forma como os indivíduos de uma espécie de distribuem em uma comunidade é um dos primeiros passos para se entender a dinâmica de populações vegetais (SILVA et al., 2012).

Para CZELUSNIAK et al. (2012) é preciso considerar que os recursos naturais uma vez extintos, estarão indisponíveis às futuras gerações. Entre estes, pode-se considerar o recurso terapêutico, pouco investigado na flora nativa, demonstrando a importância do estudo de espécies como Metrodorea flavida K. Krause, pertencente à família Rutaceae, considerada clímax, presente em ambientes de regeneração como clareiras, porém, mais adaptada a ambientes de sombra, mencionada em diversos inventários na Amazônia (SALOMÃO et al., 1995; CABRAL, 2010; CARIM, et al., 2013). Entretanto, sem estudos específicos para a estrutura populacional ou análise fitoquímica da espécie, embora apresente relevante interesse econômico, médico e cultural (CRUS, 2013).

Família Rutaceae essencialmente pantropical, apresenta cerca de 150 gêneros e 1.600 espécies, principalmente abundante nos trópicos e subtrópicos. $\mathrm{Na}$ região neotropical ocorrem cerca de 52 gêneros e no Brasil 32 gêneros nativos, incluindo gênero Metrodorea, possui oito espécies, todas nativas do Brasil (PIRANI, 2005; FERREIRA et al., 2006).

Nos vegetais, os compostos químicos oriundos do metabolismo secundário auxiliam no sistema de defesa da planta (BOSQUEIRO, 1995; CASTEJON, 2011), representando uma série de importantes princípios ativos úteis para o tratamento e cura de muitas doenças (COUTINHO et al., 2009; SILVA et al., 2010).

As análises fitoquímicas são importantes como subsídios para a farmacologia na busca de princípios ativos e na identificação de compostos bioativos, sendo que 
esses podem variar em sua composição e quantidade em função de fatores ambientais. Estas análises fornecem informações relevantes à cerca da presença de metabólitos secundários como taninos, flavonóides, saponinas e alcalóides nas plantas, permitindo supor uma atividade farmacológica em potencial para a espécie, instigando futuras pesquisas para chegar ao isolamento de princípios ativos importantes na produção de novos fitoterápicos (SILVA et al., 2010).

Neste contexto, o trabalho teve como objetivo avaliar a estrutura populacional e a distribuição espacial de Metrodorea flavida em dois fragmentos florestais do município de Alta Floresta, além de analisar qualitativamente a presença de metabólitos secundários.

\section{MATERIAL E MÉTODOS}

\section{Área de estudo}

O estudo foi realizado em dois fragmentos florestais do município de Alta Floresta-MT (Figura 1). Situado a $819 \mathrm{~km}$ ao norte de Cuiabá-MT, seu território compreende a Amazônia mato-grossense, pertencendo à Bacia Hidrográfica Amazônica, onde se destacam os rios Teles Pires, Santa Helena e Paranaíta (SEPLAN, 2014).

O clima do município pode ser considerado Am pela classificação de Köppen, apresentando temperatura média anual de $27,6^{\circ} \mathrm{C}$ e co $\mathrm{m}$ grande quantidade e distribuição de chuvas (ALVARES et al., 2014). Os solos predominantes no município são os solos Argissolos e Litólicos (SEPLAN, 2001). O tipo vegetacional é Floresta Ombrófila Densa, apresentando também porções de Floresta Ombrófila aberta, pois faz parte do sul da Bacia Amazônica (IBGE, 2012).

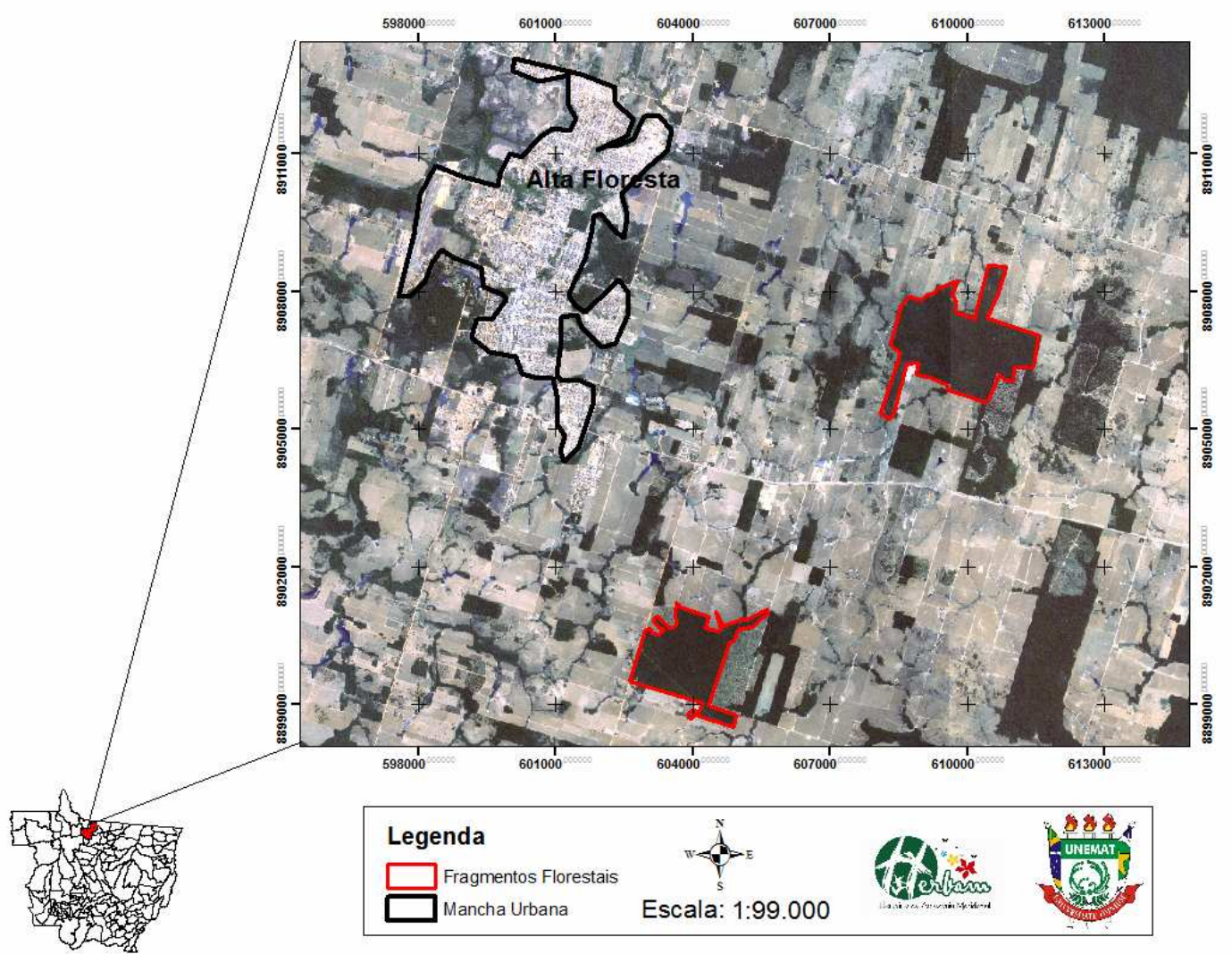

FIGURA 1 - Localização dos fragmentos florestais estudados, Alta Floresta, MT. 


\section{Amostragem e análises estruturais}

Para a amostragem da população de $M$. flavida foram estabelecidas 10 parcelas de $10 \times 100 \mathrm{~m}$, sendo $5 \mathrm{em}$ cada fragmento, totalizando 1 ha de área amostral. Dentro das parcelas todos os indivíduos com DAP $\geq 9,54 \mathrm{~cm}$ passaram pelo processo de identificação e foram marcados com plaquetas de alumínio, sendo mensurados os dados de altura total e diâmetro.

Para correta identificação da espécie, foram coletadas amostras férteis de acordo com os métodos de FIDALGO \& BONONI (1984), e as coletas foram prensadas e as exsicatas confeccionadas conforme as orientações do Manual Técnico da Vegetação Brasileira (IBGE, 2012). A identificação foi de acordo com APG III (CHASE \& REVEAL, 2009). A revisão da nomenclatura taxonômica foi a partir da Lista de Espécies da Flora do Brasil (FORZZA et al., 2015) e o material coletado foi incorporado ao acervo do HERBAM (Herbário da Amazônia Meridional UNEMAT). Os parâmetros estruturais avaliados foram densidade, frequência, área basal, sendo que para o processamento dos dados foi empregado o software FITOPAC 2.1.

Para melhor entendimento da estrutura de $M$. flavida na área, foi elaborado um histograma de frequência de classes de diâmetro e de altura. Onde o intervalo de classes foi obtido mediante cálculos matemáticos pelo modelo de Sturges (STURGES, 1926 citado por COSTA et al., 2010), caracterizado pela expressão $\mathrm{h}=$ $\mathrm{H} / \mathrm{K}$, onde $\mathrm{K}$ é o número de classes obtido por $\mathrm{K}=1+3,3^{*} \log (\mathrm{n})$ em que: $\mathrm{n}=$ número de elementos da amostra e $\mathrm{H}$ é amplitude total, calculada a partir da diferença entre o valor maior e o valor menor de altura e diâmetro dos indivíduos amostrados. Através do cálculo foi definido 7 classes para as variáveis de altura e diâmetro, representado na Tabela 1.

TABELA 1: Classes de diâmetro e altura definidas pelo modelo de Sturges.

\begin{tabular}{cll}
\hline Classe & Intervalos de Altura $(\mathrm{m})$ & Intervalos de Diâmetro $(\mathrm{cm})$ \\
\hline 1 & $<6,0$ & $<11,7$ \\
2 & $6,0-8,1$ & $11,7-13,9$ \\
3 & $8,1-10,1$ & $13,9-16,1$ \\
4 & $10,1-12,2$ & $16,1-18,3$ \\
5 & $12,2-14,2$ & $18,3-20,5$ \\
6 & $14,2-16,3$ & $20,5-22,7$ \\
7 & $>16,3$ & $>22,7$ \\
\hline
\end{tabular}

\section{Distribuição espacial}

Para avaliar a distribuição espacial da população foi utilizado o índice de PAYANDEH (1970), conforme descrito por DURIGAN (2004), caracterizado pela expressão:

$$
\mathrm{P}=\mathrm{V} / \mathrm{P}
$$

Onde: $\mathrm{P}$ é o índice de agregação; $\mathrm{V}$ é a variância do número de plantas por parcela; e M é a média do número de indivíduos por parcela. Sendo que os valores de $P<1,0$ indicam distribuição regular; $P$ entre 1,0 e 1,5 indica distribuição aleatória; e $P>$ que 1,5 indica distribuição agregada. 


\section{Análise fitoquímica}

Para a triagem fitoquímica foram coletadas amostras de folhas de $M$. flavida nos dois fragmentos. As folhas de cada fragmento foram secas separadamente à $40^{\circ} \mathrm{C}$, e posteriormente, moídas para a preparação dos extratos. Para a preparação dos extratos, de cada fragmento, adicionou-se $100 \mathrm{~mL}$ de etanol 92,8\% e 20 gramas de folha seca moídas. A solução foi filtrada após permanecer 15 minutos em banhomaria.

A triagem fitoquímica foi realizada pela metodologia de Prospecção Preliminar conforme descrito por MATOS (1997), sendo realizados testes nos extratos hidroetanólicos para a classe de taninos, alcalóides, flavonóides e saponinas, com reagentes específicos para cada substância pesquisada (Tabela 2). Os testes foram considerados positivos por reações de precipitações, coloração e/ou formação de espuma.

$\mathrm{Na}$ descrição dos resultados foi utilizado o sistema de cruzes (GARCIA et al., 2003) para especificar a presença ou ausência dos metabólitos, cujos critérios foram: presença substancial ${ }^{(++)}$, presença notável ${ }^{(++)}$, presença leve ${ }^{(+)}$e ausência $(-)$.

TAELA 2: Substâncias utilizadas para Prospecção dos Constituintes da Planta

\begin{tabular}{ll}
\hline Metabolismo & Reagente do Ensaio \\
\hline Flavonóide & Fita magnésio e $\mathrm{HCl}$ \\
Alcalóide & Dragendorff \\
Tanino & $\mathrm{FeCl}_{3}$ \\
Saponina & $\mathrm{Clorofórmio}$ \\
\hline
\end{tabular}

\section{RESULTADOS E DISCUSSÃO}

Nas áreas estudadas foram encontrados 58 indivíduos de $M$. flavida, com densidade de 84 e 32 ind. ha- ${ }^{1}$, nos fragmentos I e II, respectivamente, sendo que todas as parcelas apresentaram pelo menos um indivíduo, indicando alta freqüência das espécies nos remanescentes. Os valores de densidade do fragmento II, foi próximo ao encontrado por CABRAL (2010), em um fragmento urbano na Amazônia mato-grossense e maior que o encontrado por CARIM et al. (2013) em uma área no Amapá, já a densidade do Fragmento I foi consideravelmente maior que nos dois trabalhos mencionados anteriormente.

Os indivíduos apresentaram diâmetro médio de 14,9 cm, e área basal de 0,79 $\mathrm{m}^{2} /$ ha, no fragmento I, e $12,3 \mathrm{~cm}$ e $0,21 \mathrm{~m}^{2} / \mathrm{ha}$, no fragmento II, sendo que em ambas as áreas, o maior número de indivíduos concentrou-se principalmente nas três primeiras classes de diâmetro com cerca de 69 e 93\% dos indivíduos, fragmento I e II, respectivamente. A distribuição dos diâmetros apresentou o padrão J invertido, ou seja, alta concentração de indivíduos nas classes menores e redução acentuada no sentido das classes maiores (Figura 2), o que ocorre normalmente em populações naturais. SOUZA et al. (2009) ressaltam que esse padrão auxilia na regeneração dos ambientes fragmentados, como no caso do ambiente estudado. 


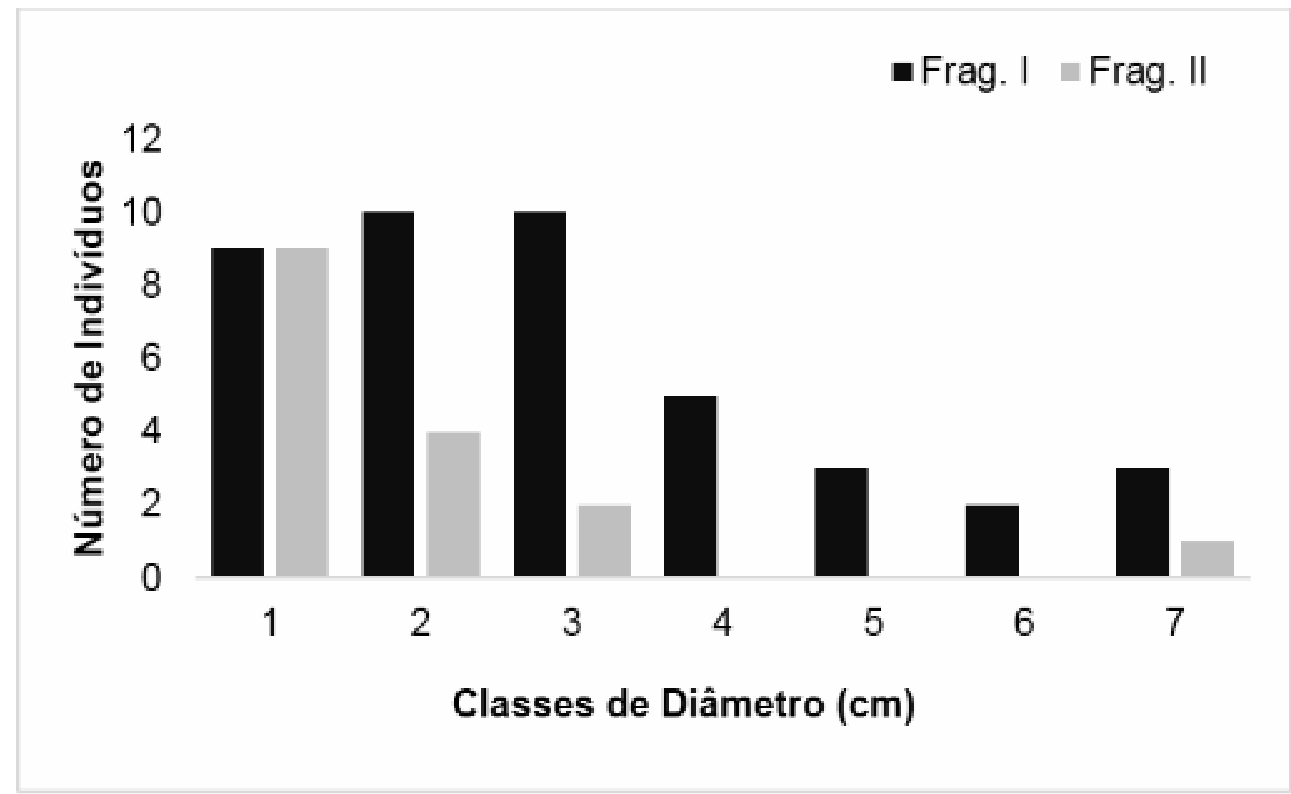

FIGURA 2 - Distribuição dos indivíduos por classe de diâmetro, nos dois fragmentos em estudo.

A altura média dos indivíduos foi semelhante em ambos os fragmentos ficando em torno de $9 \mathrm{~m}$. O fragmento I apresentou cerca de $69 \%$ dos indivíduos nas três primeiras classes de altura. Já o fragmento II apresentou todos os indivíduos em alturas intermediárias entre 6 e 12,2 m. A distribuição dos indivíduos nas classes de altura indica que a maioria dos indivíduos estão concentrados em alturas intermediárias em ambos os fragmentos, como representado na Figura 3.

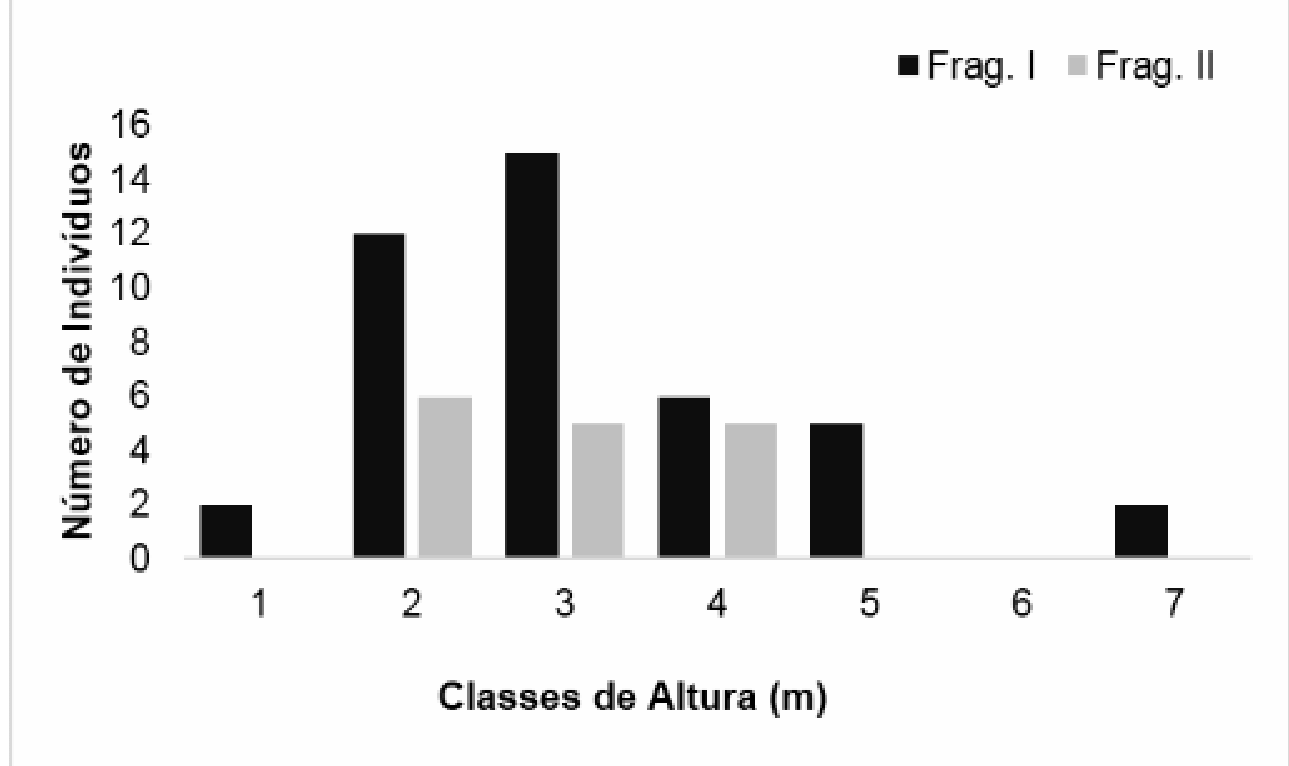

FIGURA 3 - Distribuição dos indivíduos por classe de altura, nos dois fragmentos em estudo. 
O padrão da distribuição dos indivíduos de $M$. flavida nas classes de diâmetro e altura em ambos os fragmentos demonstra uma população com muitos indivíduos de pequeno porte e finos, sendo poucos indivíduos de maior porte e diâmetro, indicando que a espécie possui um estoque de árvores finas que permitirão a substituição das árvores de maior porte que possam ser eliminadas dos ambientes estudados. Estudos da região amazônica consideram a espécie $M$. flavida como sendo de pequeno porte (SALOMÃO et al., 1995) que raramente atingem $40 \mathrm{~cm}$ de DAP (SALOMÃO \& ROSA, 1989), como apresentado nesse estudo.

O índice de Payandeh foi de 0,63 para o fragmento I e 1,15 para o fragmento II, indicando respectivamente distribuição espacial regular e aleatório. Essa diferença na distribuição se deve provavelmente a distinção do número de indivíduos entre as parcelas amostradas em cada fragmento, bem como no tamanho e no grau de conservação de cada fragmento, uma vez que o fragmento I é menor e apresenta maior grau de fragmentação do que o fragmento II. Comparando os remanescentes in loco, nota-se que o fragmento I, apresenta-se com maior número de clareiras quando comparado ao fragmento II, o que favorece diferenças locais nas condições ambientais de microclima, luminosidade, umidade, solos, fatores determinantes no padrão de distribuição das espécies vegetais (BERNASOL \& LIMA-RIBEIRO, 2010). As diferentes condições ambientais nos fragmentos, pode explicar a diferença encontradas entre os fragmentos, relacionados a distribuição da estrutura vertical e horizontal para as populações estudadas.

O padrão de distribuição uniforme tende a ocorrer em ambientes com recursos limitados, onde, devido a saturação dos sítios disponível, ocorre severa competição. Já o padrão aleatório pode implicar homogeneidade ambiental ou padrões comportamentais não seletivos (MATTEUCCI \& COLMA 1982, citado por SILVA et al., 2012).

No fragmento I, os indivíduos ocorreram com semelhante abundância nas parcelas, com exceção da parcela 1, com 12 indivíduos, o que contribuiu para a uniformidade de espaçamento entre os indivíduos. No fragmento II, embora tenham sido encontrados indivíduos em todas as parcelas, a abundância é diferente entre essas, ocorrendo desde seis indivíduos na parcela um a um indivíduo na parcela quatro, contribuindo para a aleatoriedade dos indivíduos (Tabela 3).

TABELA 2 Dados estruturais de M. flavida nos fragmentos por parcelas.

\begin{tabular}{ccccccc}
\hline Fragmento & Parcela & № Ind & AM & DM & Vol & AB \\
\hline \multirow{4}{*}{ I } & 1 & 12 & 11,08 & 17,30 & 0,220 & 0,30 \\
& 2 & 6 & 9,67 & 13,98 & 0,092 & 0,10 \\
& 3 & 8 & 10,25 & 14,13 & 0,152 & 0,13 \\
& 4 & 7 & 8,41 & 14,53 & 0,131 & 0,13 \\
II & 5 & 9 & 8,30 & 13,55 & 0,058 & 0,13 \\
\hline & 1 & 6 & 10,25 & 13,79 & 1,080 & 0,10 \\
& 2 & 2 & 9,25 & 12,72 & 0,230 & 0,03 \\
& 3 & 4 & 8,13 & 11,56 & 0,350 & 0,04 \\
& 4 & 1 & 8,00 & 12,25 & 0,090 & 0,01 \\
& 5 & 3 & 8,20 & 10,49 & 0,220 & 0,03 \\
\hline
\end{tabular}

Em que: Altura média-AM (m); Diâmetro médio-DM (cm); Volume-Vol (m³); e Área basal-AB $\left(m^{2}\right)$. 
Os dados estruturais apresentam a parcela 1 do fragmento I com a maior densidade e consequentemente, maiores valores de altura média, diâmetro médio, volume e área basal. Contudo, a parcela 4, apresentou o segundo maior diâmetro médio, enquanto a parcela 3 apresentou o segundo maior volume. No fragmento II, observa-se os maiores valores estruturais para a parcela 1 que apresentou a maior abundância da referida espécie. A parcela 2 apresentou o segundo maior diâmetro médio, e a parcela 3 o segundo maior volume para o fragmento II.

Quando comparados, os dados estruturais de cada fragmento, observa-se que, embora o fragmento II tenha apresentado menor número de indivíduos, os valores estruturais são próximos dos valores encontrados para o fragmento I, com maior número de indivíduos. Com isso pode-se dizer que $M$. flavida é uma espécie que se estabelece em áreas de clareiras, com tendência a ter elevado número de indivíduos nesses ambientes de maior luminosidade, já que estas condições favorecem o desenvolvimento desses indivíduos e a competição entre os mesmos. Contudo, sendo uma espécie adaptada à ambientes sombreados, os indivíduos que se estabelecem nesses ambientes tendem a investir em diâmetro. Na parcela 4 do fragmento II, um único indivíduo apresentou o terceiro maior diâmetro médio do fragmento. Além disso, o menor número de indivíduos de $M$. flavida pode ser explicada pela presença da mesma, próximo as clareiras presentes no fragmento II.

$\mathrm{Na}$ Tabela 3 é apresentado o comportamento dos compostos secundários avaliados mediante análise qualitativa. Os ensaios de flavonóides no fragmento II foram positivos. Já para o fragmento I o teste apresentou coloração negativa. As classes de taninos, saponinas e alcalóides também foi positivo em $M$. flavida, para ambos os fragmentos. Entretanto, no fragmento II, a espécie apresentou presença notável desses metabólitos quando comparado ao fragmento I. Esta variação pode ser decorrente das diferenças abióticas, entre os remanescentes, como condições de solo e clima (SANTOS \& BLATT, 1998), como já discutido anteriormente.

TABELA 3: Análise qualitativa das classes de compostos secundário de M. flavida nos dois fragmentos florestais.

\begin{tabular}{|c|c|c|}
\hline Classe de metabólito & Fragmento I & Fragmento II \\
\hline Flavonóide & - & ++ \\
\hline Tanino & + & +++ \\
\hline Saponina & + & ++ \\
\hline Alcalóide & + & ++ \\
\hline
\end{tabular}

O resultado positivo de alcalóide corrobora com o estudo de Baetas et al. (1999) para a $M$. flavida. Resultados positivos de alcalóide e flavonoide também foram encontrados em outras espécies da família Rutaceae como Euxylophora paraensis (ISIDORO et al., 2012) e Zanthoxylum rhoifolium (KRAUSE et al., 2013). Já GARCIA et al. (2003), mencionam que os compostos secundários analisados neste estudo são bastante comuns no reino vegetal, detectando os quatro metabólitos em Morus alba.

Diversos estudos demonstram a atividade dos metabolismos encontrados em M. flavida como sendo responsáveis por atividades farmacológicas. Os flavonóides apresentam-se uso potencial frente aos processos inflamatórios, antioxidante e antiproliferativo, dentre outros (MIDDLETON et al., 2000; COUTINHO et al., 2009; 
FLAMBÓ, 2013). Os alcalóides também foram registrados com anti-inflamatórios e antioxidante (RAMSEWAK et al., 1999).

Os taninos com presença substancial em $M$. flavida evidencia importante ação anti-inflamatória, atividade antioxidante, efeito anti-microbiano, na reparação de tecidos, regulação enzimática e protéica, entre outros (CASTEJON, 2011). Já as saponinas com atividade antiinflamatória, antifúngica, antibacteriana, antimicrobiana, antiparasitária, citotóxica e antitumoral, antiviral entre outras (SPARG et al., 2004; CASTEJON, 2011).

PRUDENTE et al., (2013), relatam a função de espécies da família Rutaceae como Citrus sinensis, Citrus aurantiifolia e Ruta graveolens no combate a doenças respiratórias, assim como sobre a ação anti-inflamatória e anti-bacteriana das espécies, principalmente devido a presença de flavonoides, estas plantas causam um efeito anti-inflamatório, através da inibição de mediadores pró-inflamatórios iNOS e COX-2; e atividade anti-bacteriana, analisada por meio do método de difusão em disco. O mesmo autor menciona que o uso de espécies medicinais da família Rutaceae para tratamento de doenças respiratórias é intenso, e que ainda existem poucos estudos que evidenciem cientificamente as ações farmacológicas, justificando assim a grande necessidade de estudos clínicos para tratamentos feitos com a utilização de espécies potenciais.

\section{CONCLUSÕES}

M. flavida em ambos os fragmentos apresentou elevado número de indivíduos com menor diâmetro e altura intermediária, podendo indicar estabilidade da espécie nesse ambiente. A população apresentou-se tanto com indivíduos espaçados regularmente, quanto com distribuição aleatória.

Através dos testes de triagem fitoquímica, foi possível avaliar qualitativamente as classes de compostos presentes na $M$. flavida, o que pode sugerir seu potencial uso farmacológico, devido ao fato de ser conhecido cientificamente a indicação terapêutica de todas as classes. Portanto, fica evidente a importância da manutenção de ambos os fragmentos para a conservação desta população, bem como da comunidade vegetal como um todo.

\section{AGRADECIMENTOS}

Á Universidade do Estado de Mato Grosso pela concessão de bolsa, e ao Herbário da Amazônia Meridional (HERBAM) - UNEMAT por apoiar a pesquisa.

\section{REFERÊNCIAS}

ALVARES, C. A.; STAPE, J. L.; SENTELHAS, P. C.; GONÇALVES, J. L. M.; SPAROVEK, G. Köppen's climate classification map for Brazil. Meteorologische Zeitschrift, v. 22, n. 6, p. 711-728, 2014.

BAETAS, A. C. S.; ARRUDA, M. S. P.; MULLER, A. H.; ARRUDA, A. C.. Coumarins and Alkaloids from the Stems of Metrodorea Flavida. Journal of the Brazilian Chemical Society. v.10, n.3, p. 181-183, 1999. 
BERNASOL, W. P.; LIMA-RIBEIRO, M. S. Estrutura espacial e diamétrica de espécies arbóreas e seus condicionantes em um fragmento de cerrado sentido restrito no sudoeste goiano. Hoehnea. v. 37, n. 2, p. 181-198, 2010.

BOSQUEIRO, A. L. D. Metabólitos Secundários em plantas. Revista ciência e educação. v. 13 n. 2, p. 91-96, 1995.

CABRAL, F. F. Levantamento Florístico e Fitossociológico do "Parque Ecológico Municipal Leopoldo Linhares Fernandes", Alta Floresta, Mato Grosso, Amazônia Meridional, Brasil. 2010. 121 f. Monografia (Licenciatura em ciências biológicas) - Universidade do estado de Mato Grosso, Alta Floresta, 2010.

CARIM, M. J. V.; GUILLAUMET, J. L. B.; GUIMARÃES, J. R. S.; TOSTES, L. C. L. Composição e Estrutura de Floresta Ombrófila Densa do extremo Norte do Estado do Amapá, Brasil. Macapá, v. 3, n. 2, p. 1-10, 2013.

CASTEJON, F. V. Taninos e saponinas. 2011. 26 f. Seminários aplicados (Pósgraduação). Universidade Federal de Goiás. Goiânia, 2011.

CHASE, M.W. \& REVEAL, J.L.A. An update of the Angiosperm Phylogeny Group classification for the orders and families of flowering plants: APG III. Botanical Journal of the Linnean Society, v. 161, p. 105-121, 2009.

COSTA, C. P.; CUNHA, C. N.; COSTA, S. C. Caracterização da flora e estrutura do estrato arbustivo-arbóreo de um cerrado no Pantanal de Poconé, MT. Biota Neotropica. v.10, n.3, p. 61-73, 2010.

COUTINHO, M. A. S.; MUZITANO, M. F.; COSTA, S. S. Flavonoides: Potenciais Agentes Terapêuticos para o Processo Inflamatório. Revista Virtual de Química, v. 1, n. 3, p. 241-256, 2009.

CRUS, E. S. Caracteres morfoanatômicos e antogenéticos foliares de Metrodorea A. St. Hil. e espécies relacionadas (Rutaceae) sob uma pesrpequitiva filogenética. 2013. $110 \mathrm{f}$. Dissertação (Mestrado). Instituto de biociência da universidade de São Paulo. São Paulo, 2013.

CZELUSNIAK, K.E.; BROCCO, A.; PEREIRA, D.F.; FREITAS, G.B.L. Farmacobotânica, fitoquímica e farmacologia do Guaco: revisão considerando Mikania glomerata Sprengel e Mikania laevigata Schulyz Bip. ex Baker. Revista Brasileira Plantas Medicinais, Botucatu, v.14, n.2, p. 400-409, 2012.

DURINGAN, G. Métodos para análise da vegetação. In: CULLEN, L.; VALADARESPADUA, C.; RUDRAN, R. Métodos de estudo em biologia da conservação e manejo da vida silvestre. Ed. UFPR, Curitiba, 2004. 667 p.

FERREIRA, L. A.; PAULA, V. F.; CORREIA, S. J. CONSTITUINTES QUÍMICOS DA RAIZ DE Metrodorea maracasana Kaastra (RUTACEAE). In: 58 ${ }^{\mathrm{a}}$ Reunião Anual da SBPC. Anais... Universidade Estadual do Sudoeste da Bahia Florianópolis, SC, 2006.

FIDALGO, O.; BONONI, V. L. Técnicas de coleta, preservação e herborização de material botânico. Instituto de botânica. Manual, n. 4. São Paulo, 1984. 61 p. 
FLAMBÓ, D. F. A. L. Atividades Biológicas dos Flavonoides: Atividade Antimicrobiana. 2013. 31 f. Dissertação (Mestrado) Universidade Fernando Pessoa. Porto, 2013.

FORZZA, R.C.; STEHMANN, J.R.; NADRUZ, M.; COSTA, A.; CARVALHO, A.A.; WALTER, B.M.T.; BICUDO, C.; MOURA, C.W.N.; ZAPPI, D.; COSTA, D.P.; PERALTA, D.F.; LLERAS, E.; MARTINELLI, G.; LIMA, H.C.; PRADO, J.; BAUMGRATZ, J.F.A.; PIRANI, J.R.; SYLVESTRE, L. S.; MAIA, L.C.; LOHMANN, L.G.; PAGANUCCI, L.; ALVES, M.V.S.; SILVEIRA, M.; MAMEDE, M.C. H.; BASTOS, M.N.C.; MORIM, M.P.; BARBOSA, M.R.; MENEZES, M.; SOARES, M.L.; EVANGELISTA, P.H.L.; GOLDENBERG, R.; SECCO, R.; RODRIGUES, R.S.; CAVALCANTI, T.; SOUZA, V.C. Lista de Espécies da Flora do Brasil. Jardim Botânico do Rio de Janeiro. Disponível em: <http://floradobrasil.jbrj.gov.br/>. Acesso em: 20 de Ago. de 2015.

IBGE. Instituto Brasileiro de Geografia e Estatística. Manual técnico da vegetação brasileira. Série Manuais Técnicos em Geociências, n. 1. Rio de Janeiro: IBGE, 2012. $275 \mathrm{p}$.

ISIDORO, M. M. SILVA, M. F. G. F.; FERNANDES, J. B.; VIEIRA, P. C.; ARRUDA, A. C.; SILVA, S. C. Fitoquímica e quimiossistemática de Euxylophora paraensis (Rutaceae). Química Nova, v. 35, n. 11, p. 2119-2124, 2012.

KRAUSE, M. S.; BONETTI, A. F.; TURNES, J. M.; DIAS, J. F. G.; MIGUEL, O. G.; DUARTE, M. R. Fitoquímica e atividades biológicas de Zanthoxylum rhoifolium LAM., Rutaceae. Visão Acadêmica, Curitiba, v.14, n.4, p. 118-127, 2013.

KUNZ, S. H.; MARTINS, S. V.; IVANAUSKAS, N. M.; STEFANELLO, D.; SILVA, E. Fitossociologia de uma área de floresta estacional perenifólia na fazenda Amoreiras, Querência, MT. Revista Árvore, v.34 n.4, p. 713-721, 2010.

MATOS F.J. Introdução à fitoquímica experimental. 2.ed. Fortaleza: Edições UFC; 1997. 141p.

MIDDLETON, E.; KANDASWAMI, C.; THEOHARIDES, T. C. The effects of plant flavonoids on mammalian cells: implications for inflammation, heart disease, and cancer. Pharmacol Rev. v. 52 n.4, p. 673-751, 2000.

PAULA, M. D; GROENEVELD J.; HUTHA, A. Tropical forest degradation and recovery in fragmented landscapes - Simulating changes in tree community, forest hydrology and carbon balance. Global Ecology and Conservation. v. 3, p. 664677, 2015.

PIRANI, J. R. Flora da Reserva Ducke, Amazonas, Brasil: Rutaceae. Rodriguésia v. 56 n. 86 , p. $189-204.2005$

PRUDENTE, R. C. C.; MOURA, R. B. Evidências científicas para a indicação popular de algumas espécies da família Rutaceae no tratamento de doenças respiratórias na região Sudeste do Brasil. Infarma: ciência farmacêutica, v. 25, n. 1, p. 24-31, 2013. 
RAMSEWAK, R. S.; NAIR, M. G.; STRASBURG, G. M.; DEWITT, D. L.; NITISS, J. L. Biologically Active Carbazole Alkaloids from Murraya koenigii. Journal of Agricultural and Food Chemistry. v. 47 n. 2, p. 444-447, 1999.

SALOMÃO, R. P. ROSA, N. A. Análise da Vegetação de Floresta Pluvial Tropical de Terra Firme, Pelo Método dos Quadrantes: Serra Norte, Carajás, PA. Acta botânica brasílica. v. 2 n. 1, p. $27-42,1989$.

SALOMÃO, R. P.; ROSA, N. A.; NEPSTAD, D. C; BAKK, A. Estrutura Diamétrica e Breve Caracterização Ecológica Econômica de 108 Espécies Arbóreas da Floresta Amazônica Brasileira I. INTERCIENCIA. v. 20, n. 1, p. 20-29, 1995.

SANTOS, M. D.; BLATT, C. T. T. Teor de flavonóides e fenóis totais em folhas de Pyrostegia venusta Miers. de mata e de cerrado. Brazilian Journal of Botany. v.21, n. 2, pp. 135-140, 1998.

SEPLAN - Secretaria de Estado de Planejamento e Coordenação Geral. Perfil do Município. Disponível em <http://www.seplan.mt.gov.br/ > Acesso em: 04 de Mar. de 2014.

SILVA, K. E.; MARTINS, S. V.; SANTOS, N. T.; RIBEIRO, C. A. A. S. Padrões espaciais de espécies arbóreas tropicais. In: MARTINS, S. V. (Org.). Ecologia de florestas tropicais do Brasil. 2a. Edição - Revista e Ampliada. 2. ed. Viçosa, MG: Editora UFV, 2012. v. 1. 371p.

SILVA, N. L. A.; MIRANDA, F. A. A.; CONCEIÇÃO, G. M. Triagem Fitoquímica de Plantas de Cerrado, da Área de Proteção Ambiental Municipal do Inhamum, Caxias, Maranhão. SCIENTIA PLENA. v. 6, n. 2, p. 1-17, 2010.

SOUZA, S. T.; FERREIRA, T. S. SILVA, A. C.; HIGUCHI, P.; PAULINO, P. S.; GOMES, J. P.; SILVA, K. M. Estrutura Populacional De Cordyline Spectabilis Kunth \& Bouche' em um Fragmento de Floresta Ombrofila Mista Montana, em Campos Novos, SC. In. IX Congresso de Ecologia do Brasil, 2009, São Lourenço. Anais... SEB - Sociedade de Ecologia do Brasil. São Lourenço-MG, p. 232-234.2009

SPARG, S.G.; LIGHAT, M.E.; VAN STADEN, J. Biological activities and distribution of plant saponins. Journal of Ethnopharmacology, v. 94, n. 2-3, p. 219-243, 2004. 